1 Universidad de Buenos Aires (UBA). Instituto de Investigaciones de la Facultad de Psicología. Becaria Postdoctoral del Consejo Nacional de Investigaciones Científicas y Técnicas (Conicet) Buenos Aires, Argentina. claudiabang@yahoo.com.ar

${ }^{2}$ Centro de Salud y Acción Comunitaria (Cesac) № 24. Hospital General de Agudos Parmenio Piñero Buenos Aires, Argentina. mavicorin@hotmail.com

\section{Analizando prácticas participativas en salud: La Semana de Las Crianzas en Villa Soldati}

\author{
Analyzing participatory practices in health: The Rearing Week in \\ Villa Soldati
}

Claudia Bang', Marcela Corin ${ }^{2}$

RESUMEN Este trabajo describe y analiza una experiencia participativa llevada adelante por un Centro de Salud ubicado en una zona marginal urbana de la Ciudad de Buenos Aires. Dicha experiencia condensa en una semana la realización de gran cantidad de actividades creativas de prevención y promoción de salud, con una planificación conjunta entre profesionales, organizaciones y vecinos del barrio. Se analizan los principales procesos participativos implicados desde la perspectiva de la Atención Primaria de la Salud Integral. Se concluye que el trabajo creativo colectivo resultante posee una gran potencia en la promoción de salud integral en el barrio, como producción de subjetividad alternativa al modelo biomédico de atención.

PALABRAS-CLAVE Participación comunitaria. Atención Primaria de Salud. Promoción de la salud. Redes comunitarias. Crianza del niño.

ABSTRACT The purpose of this paper is to describe and analyze a participative experience conducted by a Community Health Center in a marginal zone of the City of Buenos Aires. Such experience condenses in a week the realization of a large number of prevention and promotion creative activities, with a joint planning among professionals, organizations, and neighbors. Main participatory processes included in the experience are analyzed from the Integral Primary Health Care perspective. It is concluded that the resulting collective creative work has a great power in the promotion of integral health in the neighborhood, as a production of subjectivity alternative to the biomedical care model.

KEYWORDS Community participation. Primary Health Care. Health promotion. Community networks. Child rearing. 


\section{Introducción}

El inicio del siglo XXI confluye con un proceso de revitalización de la Atención Primaria de la Salud (APS) en nuestra región, proponiendo un retorno al espíritu original de la declaración de Alma-Ata desde una perspectiva integral (OPS, 2007). Diferenciándose del carácter selectivo y focalizado que tuvo la aplicación de esta estrategia durante la década anterior, la APS integral entiende a la salud como derecho humano esencial, centrándose en la necesidad de una articulación intersectorial que asegure universalidad e integralidad en los cuidados en salud y la participación comunitaria como elemento clave y esencial en prácticas de prevención y promoción de la salud (FEO, 2015).

En Argentina, este proceso ha tenido lugar en el contexto de un sistema de salud caracterizado históricamente por la segmentación y fragmentación de sus componentes. A su vez, el desarrollo de políticas y prácticas de APS integral ha convivido con un avance del modelo biomédico de atención, cuyas características principales son el biologisismo, la a-historicidad, el a-culturalismo, el individualismo, la eficacia pragmática y la orientación curativa (MENÉNDEZ, 2003). Se ha instalado así, dentro del campo de prácticas en salud, una fuerte tensión entre la tendencia hegemónica a la objetivación y la tendencia a la subjetivación (STOLKINER, 2013). Esta última es sostenida por prácticas integrales que tienden a la ampliación de derechos.

En este proceso, numerosas son las experiencias y dispositivos locales que han incorporado prácticas de promoción y prevención de salud desde una perspectiva integral en instituciones del primer nivel de atención. Muchas de ellas incorporan estrategias no convencionales e innovadoras en salud. Sin embargo, en este complejo escenario de tensiones y conflictos, estas experiencias suelen quedar poco estudiadas, aisladas e invisibilizadas tras un discurso hegemónico que prioriza prácticas biomédicas.

El objetivo de este escrito es describir una experiencia de promoción y atención de la salud llevada adelante por una institución del primer nivel de atención y analizar los principales procesos participativos implicados desde la perspectiva de Atención Primaria de Salud Integral. 'La Semana de Las Crianzas' es el nombre de este evento comunitario realizado desde hace 9 años por el Centro de Salud y Acción Comunitaria (Cesac) $\mathrm{N}^{\circ} 24$, ubicado en una zona marginal urbana del sur de la Ciudad de Buenos Aires. Esta semana condensa la realización de gran cantidad de actividades creativas planificadas durante todo el año, con una fuerte articulación entre profesionales, organizaciones y vecinos del barrio. Este trabajo realiza un acercamiento conceptual a estas prácticas incluyendo el discurso y la perspectiva de sus actores intervinientes.

Esta investigación se apoya en la rica producción teórica del movimiento de Medicina Social/Salud Colectiva Latinoamericano (STOLKINER; GÓMEZ, 2012). De este movimiento es tomada la idea de promoción de salud referida al fortalecimiento de la capacidad colectiva para lidiar con la multiplicidad de los condicionantes de la salud y la vida (CZERESNIA; FREITAS, 2006). La idea de salud remite a un objeto complejo, inserto en el proceso dinámico de salud-enfermedad-cuidados. Desde esta perspectiva, se entiende a la promoción de la salud asociada a un conjunto de valores como vida, solidaridad, equidad, democracia, ciudadanía, desarrollo, participación y asociación, entre otros. Se refiere también a una combinación de estrategias centradas en la valorización del conocimiento popular y la participación social (CARVALHO, 2008).

Desde hace más de una década la primera autora ha trabajado sobre el desarrollo e historización de políticas y procesos participativos en salud y salud mental (BANG, 2011). A partir de ese recorrido, la participación comunitaria en salud es entendida como un proceso complejo y dinámico que se da en 
una comunidad para la concreta toma de decisiones y el abordaje conjunto del propio proceso de salud-enfermedad.

Este estudio se desarrolla como parte de una investigación posdoctoral de la primera autora, cuyo objetivo general es describir y analizar dispositivos del primer nivel de atención basados en la participación comunitaria y la producción de cuidados en salud y salud mental. Durante el trabajo de campo y en conjunto con una de las coordinadoras de la organización de La Semana de Las Crianzas y actual Jefa a cargo del Cesac (segunda autora) se analizaron algunos de los principales ejes que describen la potencia de esta experiencia, lo que dio como resultado la elaboración del presente escrito.

Este trabajo pretende constituirse en un antecedente e insumo importante para la investigación e implementación de futuras prácticas participativas en promoción de la salud, y su inclusión en políticas públicas del sector.

\section{Metodología}

El presente estudio se enmarca dentro de la investigación cualitativa en salud (MINAYO, 1997) combinando diversas técnicas para la recolección, sistematización y análisis del material. El trabajo de campo se desarrolló desde la perspectiva etnográfica, siguiendo sus dos características básicas: la presencia prolongada del investigador en campo y la utilización principalmente de las técnicas de observación participante y entrevistas en profundidad (HAMMERSLEY; ATKINTON, 1994). Esta elección surge del interés por abordar estas prácticas desde la perspectiva/visión de sus actores. En este sentido, el trabajo de campo etnográfico se basa en rescatar la perspectiva nativa, que subyace y articula el conjunto de prácticas, nociones y sentidos organizados por la interpretación y actividad de los sujetos sociales.

Durante todo un año la primera autora acompañó diversas actividades grupales y comunitarias que se desarrollan semanalmente en el Cesac con inclusión de técnicas creativas, lúdicas y artísticas. Dentro de esas actividades, se acompañó todo el proceso de planificación, realización y evaluación de La Semana de Las Crianzas. Ambas autoras participaron de encuentros de planificación, reuniones de equipos profesionales, articulaciones institucionales y reuniones de programación general de actividades. También se participó del proceso de creación colectiva de algunas de las actividades comunitarias que se presentan durante dicha semana, entre ellas la creación del Murgón de la Alegría del programa de adultos y adultos mayores del Cesac.

Este trabajo incluye la perspectiva de los actores implicados a través de entrevistas en profundidad realizadas a 8 profesionales y a 6 integrantes del Murgón de la Alegría. En las entrevistas se indagó sobre la caracterización de las prácticas objeto de estudio, así como la descripción y valoración de la propia participación en dichas prácticas y en su proceso de creación colectiva.

Se describieron y analizaron dos de los principales procesos participativos comprendidos en la experiencia: el protagonizado por los equipos profesionales del Cesac en articulación con organizaciones comunitarias y el proceso de creación de la murga del grupo de adultos y adultos mayores Grupo Alegría. Para ello se utilizaron los registros realizados por cada equipo interdisciplinario, los que estuvieron centrados en una descripción de cada propuesta, su fundamentación, objetivos, contenidos, y actividades, así como el relato de su realización y evaluación posterior. Se incluyó el análisis de una breve encuesta completada por 32 profesionales, en la que se indagaron logros, aportes, dificultades, deseos y expectativas del trabajo conjunto.

El proceso de sistematización del material se realizó siguiendo dos técnicas de análisis de contenido: el análisis temático y el análisis de relaciones. El principio de recurrencia 
temática en el material de campo permitió delimitar grandes núcleos temáticos, los que fueron agrupados siguiendo los procesos de generación de categorías y articulación conceptual por tema (VALLES, 2000). Luego, se dio un ordenamiento a todo el material siguiendo los criterios de integración local e inclusiva (WEISS, 1994), para desprender las conclusiones y reflexiones finales. Se utilizaron como criterios de validez la triangulación de técnicas en el trabajo de campo y análisis de la información, y el criterio de credibilidad etnográfica (GEERTZ, 1989). En relación a los criterios éticos, esta investigación ha sido aprobada por el área institucional correspondiente (Área programática del Hospital Piñero - Nota 20/3/14) y evaluada en sus aspectos éticos por la Comisión de Evaluación del área de Psicología y Ciencias de la Educación del Consejo Nacional de Investigaciones Científicas y Técnicas (Conicet). Esta propuesta de investigación ha sido aprobada por dicho organismo, determinando la no necesidad de una evaluación por un comité de ética específico, ya que el plan de estudio no comprendió estudios farmacológicos, clínicos, quirúrgicos, epidemiológicos o psicológicos. Tampoco comprendió el uso de equipamiento médico, historias clínicas o muestras biológicas.

Asimismo, y de acuerdo a las características de la investigación, se ha seguido un proceso gradual de obtención del consentimiento informado.

\section{El escenario del despliegue creativo: el barrio y el Cesac}

El barrio Ramón Carrillo se crea hacia fines de 1990, con la finalidad de relocalizar a la población que habitaba en el albergue Warnes, en el Barrio de La Paternal. Inicialmente la población era de 7000 personas ubicadas en dos barrios: Ramón Carrillo y Villa Fátima. A partir de entonces, la población ha crecido de forma continua, con la consecuente creación de los barrios: Carrillo II, Riestra, Piletones, Los Pinos, 26 de junio, Calaza, Calacita, La Paloma, Villa La Esperanza y La Veredita. De esta forma, la población asciende a más de 25.000 habitantes (ARISTIMUÑ̃ ET AL., 2014). El componente migratorio es una de las características más significativas: en su mayoría se trata de familias provenientes de países vecinos, y en menor medida de las provincias que conforman el noroeste argentino.

Las problemáticas de salud de la población del barrio son altamente complejas. La precarización laboral, los procesos de desarraigo producto de la migración, la simultánea adaptación y resistencia a las condiciones de vida marcadas por la pobreza y la exclusión social, el aumento de la violencia verbal y/o física en las modalidades de vinculación, la dificultad para proyectar situaciones a futuro, la convivencia de varias generaciones en el mismo espacio físico, las vivencias de duelos y situaciones traumáticas constantes, son sólo algunas de las características que hacen a la complejidad de los procesos de salud/enfermedad en el barrio (BANG ET AL., 2015).

El Cesac No 24 nace con el barrio Ramón Carrillo de Villa Soldati, y comienza a trabajar con un grupo de profesionales impregnados en las prácticas de atención primaria que continúa hasta la actualidad. Dependiente del área programática del Hospital Piñero, es el centro de referencia en salud de toda esta población, el que sostiene una fuerte vinculación con su comunidad a través del trabajo asistencial y comunitario del equipo interdisciplinario de profesionales, y del grupo de promotores de salud (CORín, 2013).

Actualmente el equipo del Cesac está constituido por 42 profesionales de planta, 7 promotoras de salud y personal administrativo, 60 profesionales residentes y rotantes (medicina general y familiar, enfermería, nutrición, educación para la salud, salud mental, terapia ocupacional, pediatría, veterinaria y trabajo social), además de profesionales externos que aportan en distintos dispositivos, como psicomotricistas, 
reikistas, docentes de educación no formal y payasos terapéuticos (Grupo Puenteclown). Se trata de un centro de salud abierto a la comunidad, que cuenta con un gran número de actividades que combinan diversos recursos creativos, dando lugar a la interculturalidad (juegoteca inclusiva, murga, Reiki, huerta comunitaria, consultorio intercultural, etc.). La tendencia a la descentralización, democratización y conducción heterárquica en la institución favorece la posibilidad de catalizar motivaciones y esfuerzos del equipo de salud y la comunidad, favoreciendo el trabajo colectivo.

Figura 1. Tapiz o Wipala tejida colectivamente entre profesionales, pacientes y comunidad del Cesac, como símbolo de integración e interculturalidad (Foto: Marcela Corín)

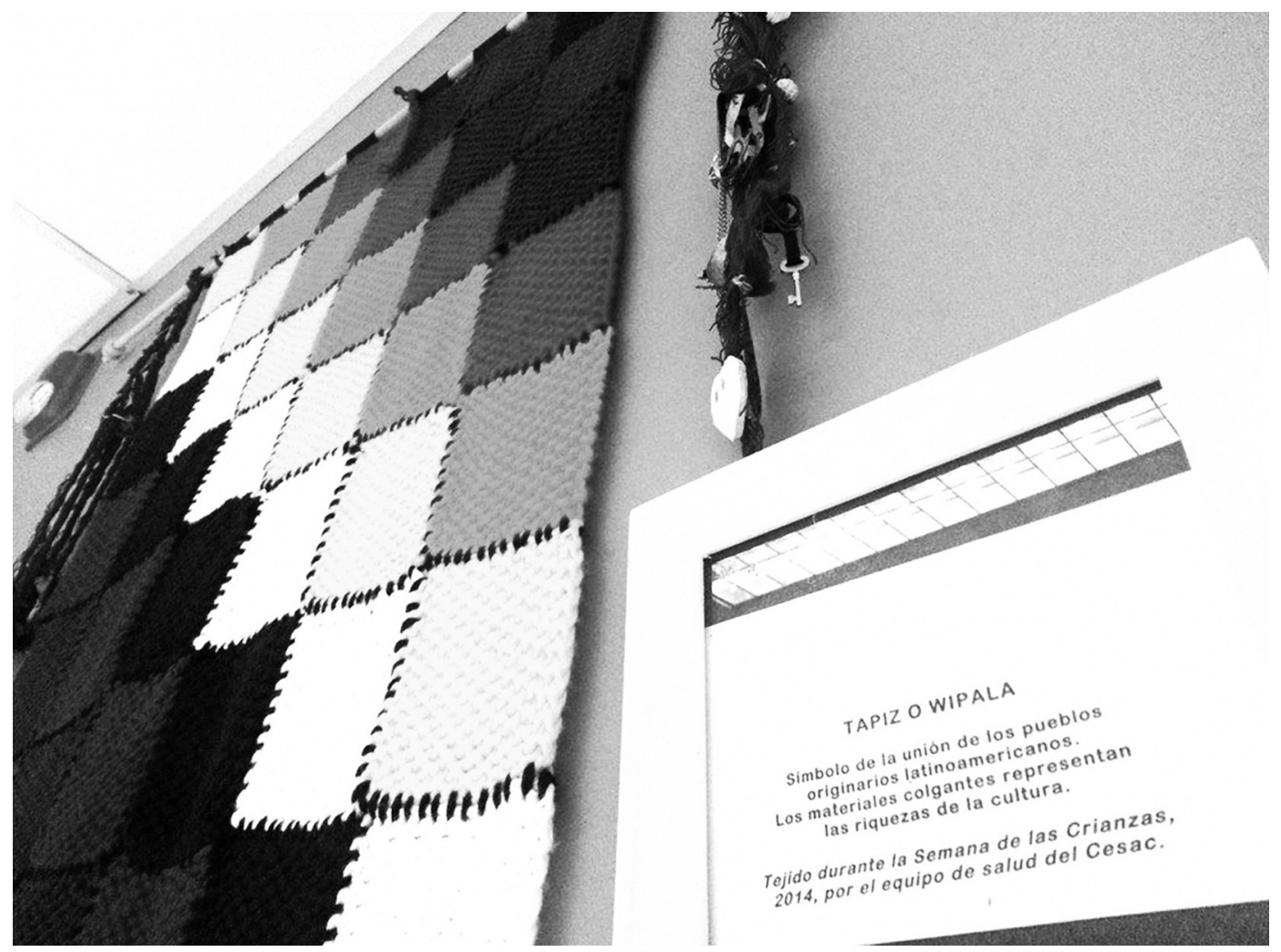

\section{La Semana de Las Crianzas}

Del registro de observación de actividades, redactado por la primera autora de este escrito, se ha elegido el siguiente relato etnográfico para comenzar la descripción de la experiencia:

En su primer jornada y ya desde muy temprano se encuentran profesionales, personal administrativo y algunos vecinos preparando la sala de espera para la inauguración de esta semana de actividades comunitarias. Algunos payasos, con sus narices rojas y divertidos guardapolvos suben altas escaleras para colgar globos y guirnaldas en los techos de salones y pasillos, mientras invitan a colaborar a todo aquel que pasa por allí. Son los Puenteclown, agrupación que se formó con la perspectiva del clown social territorial, para trabajar en centros de salud del primer nivel de atención. De a poco, la sala de espera se va colmando de gente, la que ha sido convocada por la difusión realizada durante las semanas previas: se 
trata de familias del barrio, trabajadores del Cesac, referentes comunitarios, invitados de la universidad y muchos otros. En un salón repleto de gente y luego de una breve presentación de bienvenida, el lugar se llena de música y baile a cargo de la presentación de un taller de folklore de un Centro Cultural barrial, invitado especialmente para la ocasión. Los bailarines se hacen lugar entre niños y adultos, y luego de algunas piezas de danza invitan al público a sumarse a bailar, ayudados por los payasos, quienes gustosos bailan y animan a las familias para sumarse. El momento se vive como una gran fiesta, muchos miran y se mueven en el lugar, algunos sacan fotos y otros siguen el ritmo con aplausos. Luego, se realiza la inauguración de la Juegoteca Permanente en Sala de Espera, producto de un proyecto de extensión universitaria realizado junto con la Facultad de Ciencias Sociales de la Universidad de Buenos Aires, para la incorporación de un sector acondicionado con juguetes y libros, como espacio permanente de juego para niños y niñas. En la pared de dicho sector se ve un gran mural con el dibujo de niños de diferentes procedencias, que dice: 'El rincón de la Patria Grande', lo que muestra el espíritu de inclusión e interculturalidad que atraviesa esta y todas las actividades propuestas. En dicha inauguración se invita a todos los niños y niñas presentes a conocer y jugar en este rincón. Mientras la gente conversa y sigue saludándose afectuosamente, se van acomodando tarros de pintura y pinceles para la realización de un mural colectivo en una pared externa lateral de la institución. Un grupo de muralistas junto al equipo de trabajadores y la comunidad viene realizando este mural en etapas, el que aborda la temática, también elegida colectivamente, del juego y la interculturalidad. Mientras en otra de las entradas ya se encuentra funcionando la posta de vacunación con vívidos carteles que invitan a acercarse, se preparan algunos equipos profesionales para salir a realizar diversas actividades creativas en instituciones del barrio, las que han sido planificadas durante los meses anteriores.

Figura 2. Decoración de sala de espera, momentos antes de la apertura del Cesac para la inauguración de La Semana de Las Crianzas (Foto: Claudia Bang)

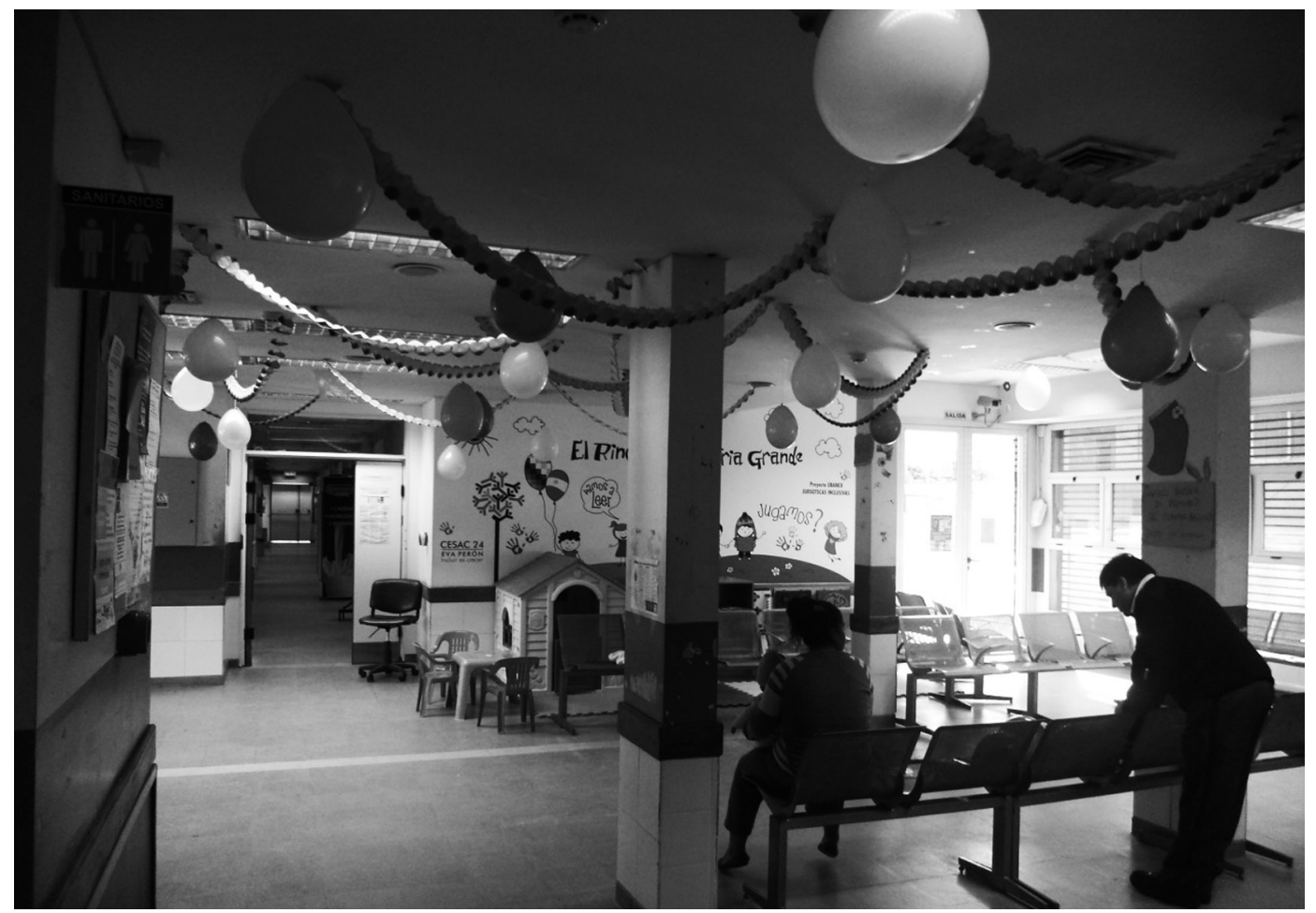


De una forma similar, cada año se da comienzo al cronograma de esta semana con la realización de múltiples actividades dentro y fuera del centro de salud: talleres, espectáculos, propuestas creativas de realización colectiva y dispositivos de prevención y promoción de la salud que incorporan recursos lúdicos, artísticos y creativos. Además, en toda la semana se realiza una gran cantidad de controles de salud y postas de vacunación.

Numerosos artistas y referentes comunitarios son invitados: algunos ejemplos son las presentaciones artísticas de Los Títeres del Piñero coordinado por el equipo interdisciplinario de gerontología del Hospital Piñero, o el programa Breves Músicas de la Dirección General de Enseñanza Artística de la Ciudad de Buenos Aires. La edición 2014 culminó con la preparación de una Apacheta (lugar sagrado y ofrenda realizada por los pueblos originarios andinos a la Pachamama) realizado junto a una chamana del barrio.

Figura 3. Ritual a la Pachamama y preparación de Apacheta en la entrada del Cesac (Foto: Marcela Corín)

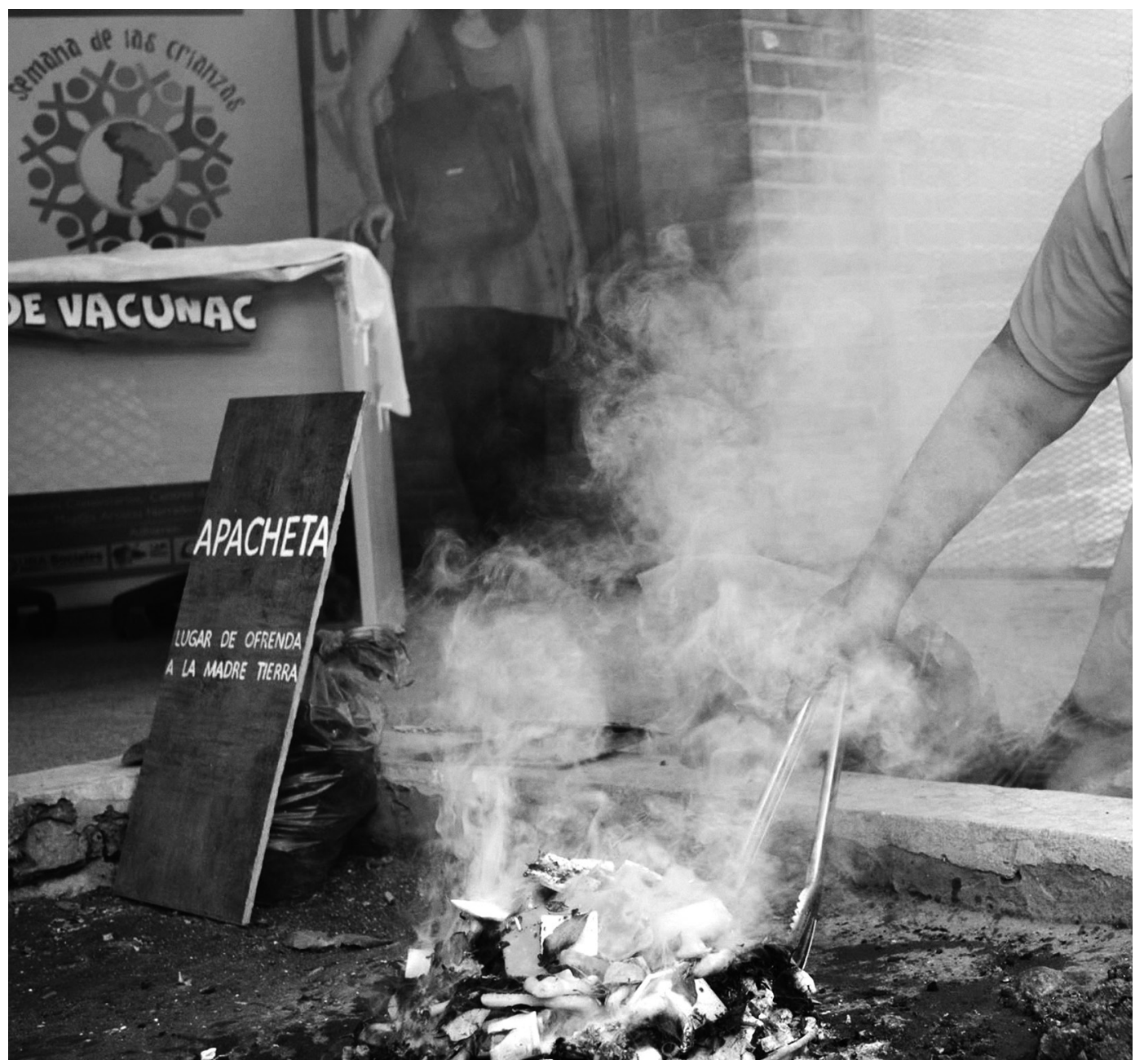


El entramado creativo resultante invita a una participación abierta, con gran visibilidad en el barrio. De esta forma, el Centro de Salud se nutre de actividades creativas y participativas para poner el foco en la necesidad de trabajar desde la salud, visibilizando los procesos de salud/enfermedad/cuidados. Así lo manifiesta una de las coordinadoras entrevistadas:

el objetivo es no trabajar sólo con la enfermedad, hacer consciente al barrio y a nosotros mismos que trabajamos para el autocuidado, para que la gente pueda comprender que puede venir acá sin estar enfermo, para otras cuestiones, para hacer actividades que les hacen bien.

La historia de La Semana de Las Crianzas comenzó en 2009, inspirada en la experiencia de la Semana del Bebé realizada por Salvador Celia en Canela, al sur de Brasil (GORODICH, 2003). A partir de una capacitación recibida en derechos de infancia y adolescencia (CRED-PRO, 2006) el equipo de profesionales del Cesac se planteó la necesidad de crear y transformar dispositivos que contemplen los derechos de los niños, y sobre todo dieran visibilidad a las acciones que se venían realizando.

Del análisis del material de campo se desprende que en la realización de estas actividades lúdico-artísticas confluyen varios procesos heterogéneos de participación comunitaria coordinados por actores diversos. Los principales procesos son:

1. Desde el año 2014 se lleva adelante un proceso de planificación conjunta de actividades entre los profesionales del Cesac en articulación con instituciones del barrio. Para ello se dispone de una reunión mensual del curso anual de postgrado dictado en la institución. La consigna es la conformación de equipos de trabajo heterogéneos que planifiquen, realicen, registren y evalúen dos actividades por grupo: una dentro de la institución y otra en una organización o institución barrial. Luego de una capacitación para la realización de actividades creativas con participación comunitaria, se disponen momentos para el trabajo grupal, para que los equipos salgan a planificar junto a las instituciones y organizaciones barriales, así como para la realización de registros. Las autoras de este escrito fueron parte de la coordinación de dicho proceso.

2. En segundo lugar, se encuentran las actividades sostenidas por los mismos trabajadores, grupos profesionales que realizan habitualmente tareas en la institución o invitados especiales. Algunos ejemplos son: un show de magia en sala de espera realizado por una trabajadora social del Cesac, la realización colectiva de un tapiz intercultural, las actividades del Grupo Puenteclown, talleres de musicoterapia y juegos para niños y adultos, entre tantos otros. En este marco y desde el año 2013 el grupo de adultos y adultos mayores Grupo Alegría que se reúne semanalmente en le Cesac ha conformado una murga.

Los escenarios de realización de estas actividades también son múltiples: se desarrollan actividades simultáneas en sala de espera, en el salón de usos múltiples y en los consultorios. Otras tantas actividades salen del Cesac para hacer un recorrido por el barrio y llegar a centros educativos, escuelas, Organizaciones No Gubernamentales (ONGs) y comedores, entre otros. Esta articulación posibilita la circulación de los profesionales, vecinos y otros participantes por diferentes espacios significativos del barrio.

Los controles de salud se potencian y cuadruplican durante esa semana, alcanzando los 200 a 270 controles realizados por las pediatras y médicas generalistas. Las postas de vacunación se realizan en la puerta del Cesac durante toda la semana, además del vacunatorio y las postas extramurales. También se pone en marcha el observatorio de desarrollo infantil interdisciplinario en consultorios conjuntos, 
probando distintas herramientas para el registro, seguimiento y valoración del proceso de desarrollo en los primeros años de vida.

Cabe destacar que, desde el año 2016 (luego de haberse realizado el trabajo de campo), se ha decidido realizar un proceso más amplio que incluye varias semanas de actividades en distintos momentos del año, utilizando una similar metodología de creación colectiva. Se han incorporado nuevos promotores de salud formados en el Cesac y se han incluido otras actividades a lo largo del año (Día internacional de lucha contra la diabetes, diversas campañas de prevención, entre otras). Este proceso en crecimiento constante permite que esta metodología participativa de trabajo no se vea reflejada sólo en la semana de las crianzas, sino que va nutriendo a todas las actividades que se desarrollan desde el Cesac, posibilitando una participación cada vez más amplia de vecinos, instituciones y equipos de salud.

\section{Análisis de los procesos participativos}

Dentro de los múltiples procesos participativos que conforman esta experiencia se estudiaron aquellos que han comprendido procesos sostenidos en el tiempo, donde profesionales, pacientes, referentes barriales y otros se reúnen semanal o quincenalmente durante gran parte del año para planificar, realizar y evaluar las actividades conjuntas que tienen lugar en la semana del evento. En primer lugar, se aborda el proceso que realizan los equipos profesionales del Cesac en articulación con instituciones del barrio, y luego se aborda el proceso de creación de 'El Murgón de la Alegría'. Se describen y analizan estos procesos a través del discurso de sus protagonistas: los profesionales y trabajadores del Cesac, y los adultos y adultos mayores participantes de la murga del Grupo Alegría.

\section{Sobre el proceso creativo colectivo entre profesionales $\mathrm{e}$ instituciones}

El siguiente análisis se realiza sobre la base de los registros de observación participante, los registros redactados por cada equipo de profesionales, y las entrevistas y encuestas realizadas a sus miembros.

Durante el período que duró la investigación la conformación de cada equipo resultó fuertemente interdisciplinaria, con la inclusión de profesionales del Cesac, profesionales residentes y promotores de salud. Como resultado de este proceso se realizaron 6 actividades en la institución y 6 en comedores, instituciones educativas del barrio y una radio comunitaria. Se incluyeron la realización de obras de teatro, espacios de juego inclusivo, lectura teatralizada de cuentos, la realización de una columna radial, teatro de sombras, entre otros.

Los entrevistados destacan la importancia de la realización de estas actividades, ya que, si bien tienen lugar en una sola semana, concentran gran parte del trabajo anual que compromete a muchos actores y se presenta como oportunidad para trabajar algunas temáticas de salud integral a través de procesos creativos. Todos los grupos describieron el contenido de su propuesta haciendo referencia a la inclusión de recursos lúdicos y artísticos: se las define como propuestas artísticas, lúdicas, expresivas y lúdico-educativas. Se valora muy positivamente la inclusión de recursos lúdicos, artísticos y creativos en las actividades, según una profesional entrevistada: "en relación al arte y la salud, nos sirve para buscar y encontrar otro tipo de herramientas para trabajar". Particularmente se utilizaron recursos teatrales, literarios, plásticos, musicales y lúdicos (juego libre y reglado). Durante la realización de las actividades, observamos que se fomentó la participación activa de los niños, niñas y adultos participantes, los que fueron 
invitados a leer, jugar, dibujar, utilizar títeres, y reflexionar sobre diferentes aspectos de la salud y los cuidados.

Siguiendo el criterio de recurrencia temática hemos ordenado los aspectos más significativos mencionados por los profesionales en los siguientes tres apartados:

\section{La perspectiva de derechos y la im- portancia de trabajar desde lo vincu- lar y creativo}

Las entrevistas y los registros hacen referencia en primer lugar, a que las actividades planificadas se fundamentaron en el derecho de las niñas y niños a jugar y participar en actividades recreativas que favorecen el desarrollo de su salud en forma integral, incluyendo necesidades sociales, culturales, emocionales y psicológicas. De allí se destaca la importancia de la construcción de espacios de lectura y juego desde la primera infancia, con la inclusión de estrategias creativas. Según una de las profesionales que coordinó la actividad del rincón de lectura:

pensamos que posibilitar que haya espacios creativos para los niños es apostar a la imaginación, herramienta indispensable para que un ser humano pueda desarrollarse.

Todos los registros y entrevistas coinciden en que sus propuestas han incluido activamente la mirada y acción de los niños y adultos participantes, estimulando la toma activa de la palabra. Estas actividades son entendidas como espacios subjetivantes y de expresión para los niños y niñas del barrio, al estimular el vínculo con padres, niños y otros adultos. En este sentido, los relatos de los profesionales han puesto el acento en la posibilidad de generar placer y disfrute a través de las actividades vinculares realizadas. Así reflexionaba un profesional:

la actividad pudo lograrse con sensaciones de mucho entusiasmo, creatividad, alegría, emoción, y mucha diversión... por parte de las familias que participaron y de nosotros mismos.

Según un registro grupal: "todos los profesionales del equipo de salud formamos parte de la organización generando entusiasmo y propuestas". En cuanto a la comunidad, se expresa que

es una oportunidad rica y valiosa que todos esperan en el barrio: niños y familias por una mayor equidad en salud y mayores oportunidades de juego y creatividad.

De esta forma, se entiende que las características vinculares de estas actividades refuerzan acciones de promoción y comunicación. 


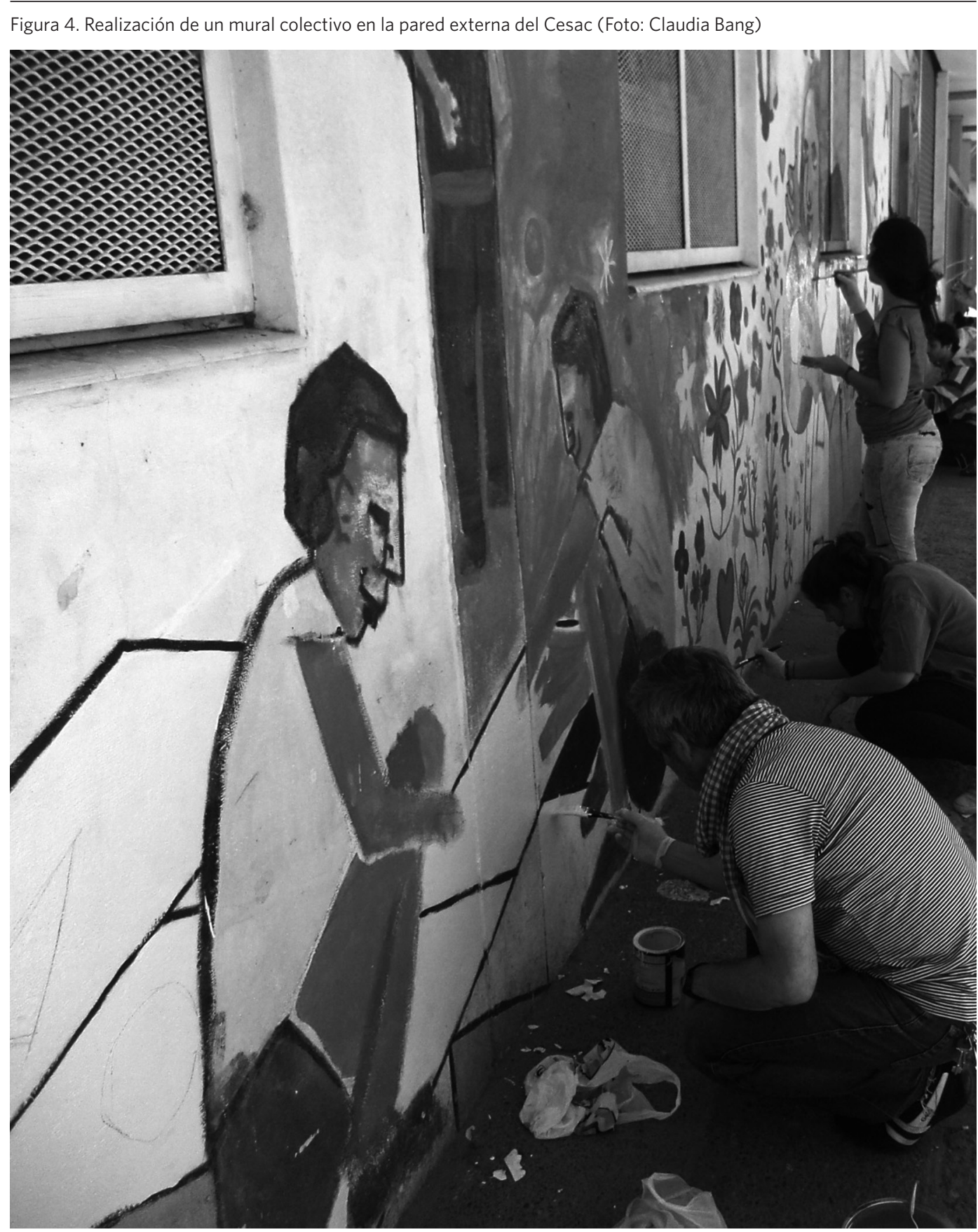

La posibilidad de conocerse y compartir saberes y prácticas en los equipos profesionales

Según lo expresado en los registros grupales, la planificación fue vivida desde el entusiasmo, destacándose la oportunidad de trabajar grupalmente. Así lo expresaban:

fue muy significativo que nos hayamos podido poner de acuerdo, nos hayamos comprometido con las diferentes tareas asignadas desde el entusiasmo y las ganas de compartir con el otro. 
Casi todos los grupos acentúan la horizontalidad en la participación, el respeto y la escucha en cada etapa del proceso. Según una profesional:

creemos que todos participamos por igual, respetándonos, cumpliendo con lo pautado, escuchándonos y sobre todo potenciando aquello que cada uno tenía para aportar, confiando en el otro, planteando sugerencias y desacuerdos cuando fue necesario.

También se valora el haber podido trabajar con compañeros con quienes no se comparte en lo cotidiano y haber intercambiado, a través de la tarea, saberes provenientes de diferentes disciplinas, saberes extradisciplinares y experiencias personales, descubriendo el conocimiento sobre arte o juego que poseen algunos integrantes, todo lo que ha enriquecido el proceso. Al decir de otra profesional:

un hecho significativo tuvo que ver con poder encontrarnos trabajando personas que por lo general no trabajamos juntos, pudiéndonos poner de acuerdo en relación con un objetivo en común y trabajar para eso.

Para muchos ha sido una primera experiencia grupal con inclusión de recursos creativos, lo que fue sentido como un desafío interesante y motivante a la vez. Asimismo, se valora la inclusión de recursos artísticos y el proceso de creación colectiva como posibilitador de una reflexión conjunta sobre prácticas de salud y crianza. En relación a la construcción de una obra teatral, una de las profesionales mencionaba: "El mismo debate en el armado del guión fue enriquecedor, tanto para conocerse, como para pensar las problemáticas de la población”.

\section{El fortalecimiento de redes institu- cionales y comunitarias}

Entre los principales objetivos registrados por los equipos para sus tareas se encuentra la creación de espacios de encuentro con las instituciones del barrio y la comunidad, así como el fortalecimiento de lazos con organizaciones y la población para la planificación conjunta de acciones de promoción y prevención en salud. Entre los aspectos que han facilitado la tarea se destaca el poder presentar algunas propuestas desde los espacios que ya se vienen articulando con instituciones del barrio. Un profesional entrevistado así lo mencionaba:

la institución la elegimos por el vínculo que tenemos desde hace años con la referente, ya que ahí funcionaba primero un comedor y luego un Centro de Primera Infancia. Nos interesó reforzar ese vínculo.

En relación al proceso de planificación conjunta, fue significativo que todos los grupos reconocen que ellos fueron quienes planificaron y llevaron la propuesta a la institución:

pudimos evaluar que durante todo el proceso creativo pensamos en la posibilidad de trabajar, diseñar, dibujar, escribir y otras actividades con la institución receptora.

En relación a una actividad realizada en una institución nueva en el barrio, un grupo mencionaba:

evaluamos que al ser un primer encuentro, y para muchos la primera experiencia creativa, la sola propuesta y aceptación por parte del Jardín, nos eximía de las actividades en conjunto.

Los equipos y profesionales entrevistados entienden que, si bien el contacto o intercambio con las instituciones fue muy valioso, en algunas oportunidades no se ha logrado una verdadera planificación conjunta de actividades, lo que se ha constituido en un fuerte desafío. 


\section{Evaluación participativa sobre el pro- pio proceso}

Hacia el final de La Semana de Las Crianzas de 2014 se realizó un taller para el equipo de Salud. Con la finalidad de poner en común logros, dificultades, deseos y expectativas sobre el trabajo conjunto realizado, se administró una pequeña encuesta, cuyas respuestas se compartieron hacia el final de la actividad. Se registraron 32 respuestas, las que se agrupan alrededor de dos ideas: por un lado, los participantes manifestaron que han aportado a la tarea conjunta un gran esfuerzo laboral relacionado con el compromiso y la voluntad; por otro lado, se encuentran muy presentes las ideas de haber aportado alegría, felicidad, y pasión, manifestando un fuerte componente afectivo asociado a la tarea diaria. La palabra que más se repite es 'ganas', relacionado en general a las ganas de aprender y trabajar. Otras ideas muy presentes como características del trabajo conjunto están relacionadas con la dedicación, el tiempo, la voluntad, la energía, la experiencia, el amor, la alegría y la escucha. De esta forma, se rescata la importante presencia del componente afectivo relacionado con lo vincular en el establecimiento de lazos entre colegas y con pacientes/personas del barrio.

\section{Sobre el proceso creativo del Murgón de la Alegría}

Este apartado tiene como finalidad abordar el proceso creativo realizado por el taller de adultos y adultos mayores, uno de los espacios abiertos a la comunidad que funciona semanalmente en el Cesac. Del trabajo con los registros de observación surge el siguiente relato redactado por la primera autora de este trabajo:

La murga del taller de adultos y adultos mayores
Grupo Alegría se presenta durante la segunda jornada de La Semana de Crianzas 2014. Ese día, la mayoría de sus integrantes llega más temprano de lo habitual, expresando mucho entusiasmo y expectativas. Comienza a prepararse la vestimenta: sobre remeras y pantalones verdes y violetas flamean flecos de pulseras y tobilleras realizadas previamente con retazos de tela. A esto se suman accesorios representativos de diferentes países: sombreros paraguayos e insignias o telas de colores. Los rostros se decoran con diseños en purpurina, y se reparten accesorios y pelucas brillantes. Se infla una gran cantidad de globos, se prepara el estandarte, se cuelgan los tambores y se realiza la última práctica de formación. Llega la hora de la presentación y luego de darse aliento a través de palabras y abrazos en ronda, la murga sale a golpe de tambor, seguida por los pasos de una coreografía que intentará sostenerse durante todo el recorrido. Casi todos los que participamos del proceso previo estamos presentes junto al equipo de coordinación del taller (conformado por profesionales del Cesac, docentes del programa Barrios en Juego y tres integrantes del Grupo Puenteclown) y unos 20 participantes estables del grupo: hombres y mujeres del barrio, cuyas edades oscilan entre los 50 y los 80 años, la mayoría extranjeros provenientes de países vecinos.

Al comenzar el recorrido el grupo muestra gran motivación, concentrándose en ajustarse a los ritmos murgueros. Muchos vecinos se asoman a sus balcones o salen a la puerta para sacar fotos o aplaudir, lo que llena de orgullo al grupo. El recorrido finaliza en un Centro de Primera Infancia de Villa Fátima, donde esperan atentos unos 40 niños pequeños, junto a sus maestras. El grupo entra bailando y reparte globos, lo que facilita la interacción. De a poco, niños y adultos hacen rondas al ritmo de los tambores. Luego, los músicos dejan que los niños toquen los tambores y se comparte música y canciones. Al finalizar la actividad y despedirse, los rostros de niños y adultos se llenan de sonrisas. 


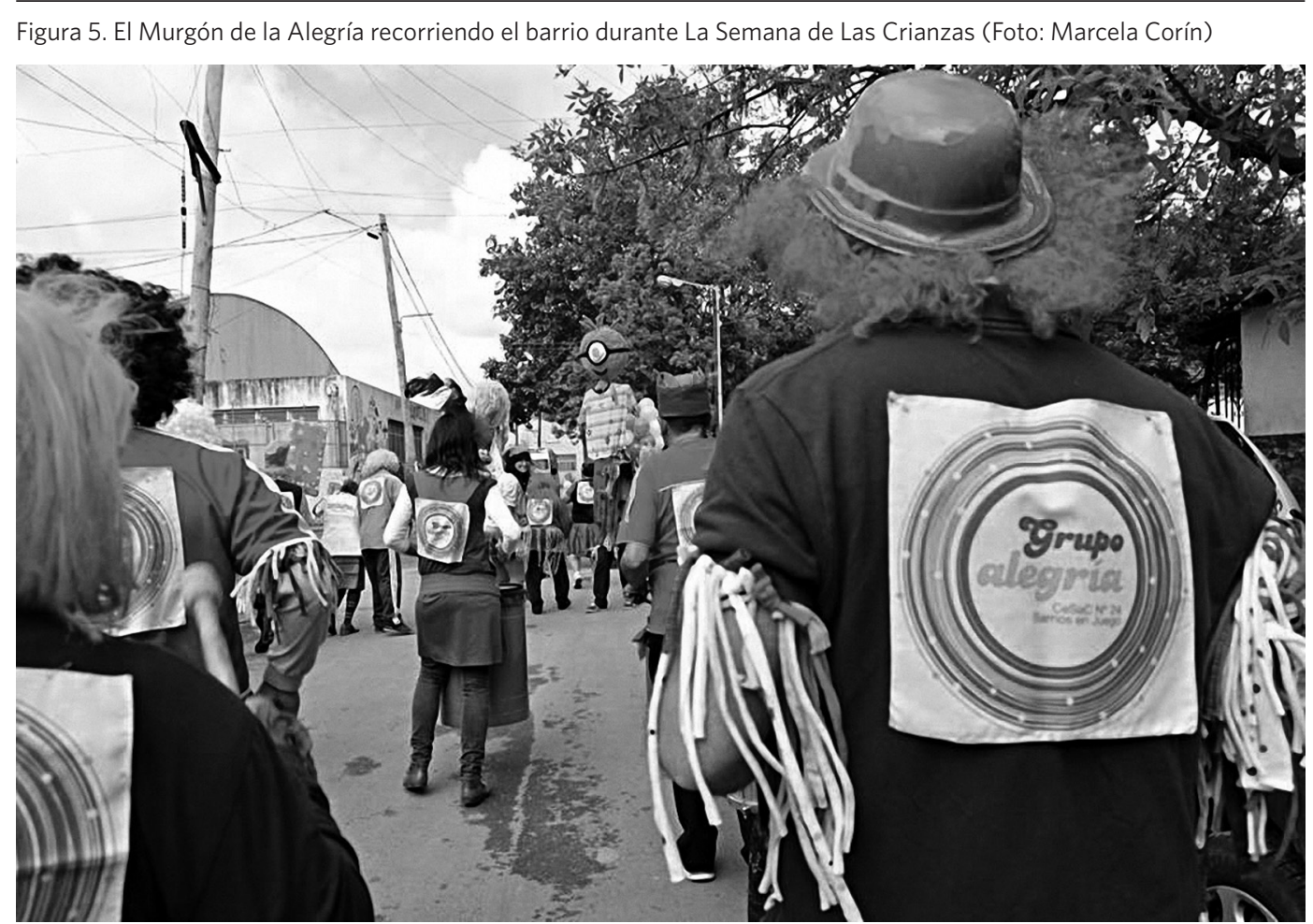

Para algunos fue la primera vez que compartían este tipo de experiencias, así lo manifestaba un integrante: "Es la primera vez que participaba. Yo nunca había estado y me pareció muy bonito estar todos juntos y compartir, divertirse y bailar todos".

El proceso de creación colectiva de esta actividad duró varios meses, en los que se destinó gran parte de los encuentros semanales para tomar decisiones colectivamente y organizar la tarea. Ya se había participado como invitados en una murga el año anterior, ahora se decidió asumir el desafío de crear colectivamente la propia murga. Para ello se inició un proceso de construir tambores con cueros y material reciclado, preparar el vestuario, ensayar los ritmos y crear la canción que acompañaría el baile, aprender los pasos y ensayar. También se decidió colectivamente el recorrido que se realizaría durante la presentación y la institución que se visitaría. Los participantes del grupo valoran haber podido realizar este proceso de forma verdaderamente colectiva y desde un rol activo. Así lo expresaba una integrante: "es que todo lo hacemos, desde el instrumento, todo hecho por nosotros mismos, no es comprado". Otro integrante mencionaba: "nosotros hicimos las cosas y uno lo hace con gusto, uno se pone y sabe que va a quedar bien, y uno así lo hace lo mejor que uno puede...” A su vez, este proceso ha reforzado un gran compromiso sostenido en lazos solidarios: "este es un proceso nuestro, porque todos compartimos, todos colaboramos. Si había lluvia igual veníamos para hacer esto".

En la evaluación colectiva posterior a la actividad muchos mencionaron haberse sentido muy a gusto y contenidos en el proceso cargado de alegría y compañerismo, como lo decía un integrante: "participando junto a los compañeros, como en familia”. En las entrevistas, muchos mencionaron que fue un gran proceso de aprendizaje: "yo aprendí cómo se hace un conjunto, cómo se hace una fiesta, cómo se organiza, y eso fue muy lindo". 
Otro participante decía: "fue bonito porque es lindo lo que hicimos, fue hermoso, porque es una cosa que aprendimos, compartimos y encima usamos". Un integrante de la batucada comentaba: "al menos a tocar el ritmo yo aprendí, porque yo no sabía tocar. Me sentíbien, nunca había tocado, yo salí músico [risas]", a lo que una compañera agregaba: "ensayando, ensayando salió y fuimos mejorando, tomando confianza". El crecimiento conjunto aparece como una consecuencia de haber pasado por este proceso: "y el grupo ha crecido, eso es lo que más me gusta, y va a crecer más y más”. Por todo lo antedicho, este proceso parece haber dejado profundas marcas en la subjetividad de todos los que formaron parte.

\section{Conclusiones}

En este recorrido nos hemos acercado a una experiencia compleja que articula diversos procesos y pone en relación a múltiples actores. Los registros y relatos analizados confluyen en caracterizar a La Semana de Las Crianzas como una experiencia fuertemente interdisciplinaria, con articulación intersectorial y gran participación comunitaria. El resultado de este proceso es la concreción de un importante entramado interinstitucional y comunitario que trabaja para la producción colectiva de salud y subjetividad en el barrio. La inclusión de una visión intercultural atraviesa toda la experiencia, dejando profundas marcas no sólo en la comunidad, sino también en los profesionales participantes.

El análisis de los procesos participativos estudiados muestra que tanto profesionales como pacientes y vecinos se sienten sujetos de una participación que se construye a través de diversos procesos de creación colectiva cargados de afectos, generando construcciones identitarias que afianzan los vínculos existentes. De esta forma, la participación comunitaria se apoya en la generación y sostenimiento de procesos, actividades, espacios y vínculos asociados al placer, lo que hemos reconocido como un componente libidinal de la participación (BANG, 2014). Se evidencia aquí lo imprescindible de la creación de dispositivos vinculares $\mathrm{y}$ afectivos que den lugar a procesos de subjetivación para el sostenimiento de procesos participativos. Se supera así una concepción instrumental y meramente racional de la idea de participación, sostenida históricamente desde las políticas y modelos normativos en Atención Primaria de Salud. La participación deja de ser aquí una colaboración pasiva a propuestas decididas por otros y sentidas como ajenas, para constituirse en verdadera estrategia de transformación conjunta de los procesos de salud/enfermedad/ atención-cuidados.

Asimismo, esta experiencia ha encontrado en los procesos de creación artística colectiva una gran capacidad para la generación de efectos comunitarios saludables ya que, como lo enuncian los entrevistados, las actividades lúdicas y artísticas, al ser abordadas desde lo colectivo, ponen en juego la imaginación, la ficción y la espontaneidad, potenciando la creatividad, componente central en el fortalecimiento de una capacidad colectiva para el abordaje de los procesos implicados en la salud y la vida.

Entendiendo a la salud desde una perspectiva integral e incorporando la lógica de derechos, estas prácticas se constituyen en potentes estrategias de promoción de salud en el ámbito comunitario. En este sentido, no es posible pensar a la salud integral separada de otros derechos como el juego, la lectura y la participación en actividades vinculares generadoras de placer (BUSTELO, 2007).

Como se ha analizado, los procesos desencadenados tienden a la conformación de espacios vinculares, posibilitadores de nuevas miradas, canalizadores de deseos y necesidades compartidos, transformadores de representaciones e imaginarios sociales, y espacio de creación colectiva que pone en juego la posibilidad de transformación de las propias 
realidades. En un contexto caracterizado por la pobreza, la exclusión social, el desarraigo y múltiples formas de violencia, estas prácticas se muestran como formas alternativas de respuesta, las que van tejiendo redes de contención institucional y comunitaria, presentando en acto otras formas de vínculos posibles.

Pero tal vez una de las dimensiones más significativas de esta experiencia está dada por su fuerte componente vincular y afectivo, muy presente en los relatos recogidos, como producción de subjetividad alternativa al modelo biomédico de atención. En oposición a un modelo centrado en la creciente objetivación de los padecimientos subjetivos (AUGSBURGUER; GERLERO, 2005), esta experiencia se muestra centrada en un 'trabajo vivo' (MERHY, 2002), posibilitado por la construcción conjunta de una vivencia compartida del hacer creativo. Por sus características lúdico-artísticas particulares y los procesos cooperativos asociados, estas prácticas permiten transmitir en acto una perspectiva de salud integral, basada en el cuidado y asociada al placer, la alegría $\mathrm{y}$ las relaciones comunitarias solidarias. El análisis de entrevistas muestra que esta experiencia ha permitido además horizontalizar vínculos entre profesionales y pacientes $\mathrm{y}$ crear conocimientos prácticos compartidos, dimensiones que Sousa Campos (2001) ha relacionado con una clínica centrada en el sujeto y en defensa de la vida.

La Semana de las Crianzas, como parte de una estrategia integral de promoción de salud, permite además visibilizar, optimizar y articular recursos existentes en la comunidad. Según Bertucelli, Lerda y Mercado (2002), este sería un modo estratégico de intervención comunitaria en salud, ya que se apoya en procesos colaborativos, articulándose al movimiento de las redes comunitarias existentes. En este sentido, la inclusión de la perspectiva intercultural posibilita el reconocimiento y la circulación de múltiples saberes y prácticas presentes en la comunidad, fortaleciendo procesos participativos, al ser congruentes con las formas del hacer comunitario.

\section{Reflexiones finales}

Este desarrollo nos pone de cara a la necesidad de potenciar estrategias y prácticas complejas de participación comunitaria que integren los elementos del proceso creativo en su contexto socio-histórico-cultural dentro de la estrategia de promoción de salud. En este sentido, la cosmovisión andina nos permite repensar las prácticas de salud a través de la idea de Buen Vivir/ Vivir Bien, como forma de vivir comunitaria en armonía con el entorno, a través de prácticas cotidianas de respeto, cooperación y solidaridad (HUANACUNI, 2010). Desde la perspectiva de salud colectiva, nos proponemos recuperar estos valores generando prácticas que confronten al individualismo y la competencia propios de la sociedad de mercado (ALAMES, 2011). Se trata también de reconocer y recuperar el placer como práctica de salud y salud mental, y como producción de subjetividad tendiente a la humanización en la atención de los padecimientos. La multiplicación de estas experiencias permitiría afianzar redes y vínculos comunitarios, favoreciendo también la expresión de la diversidad cultural presente, por ejemplo en las distintas formas de crianzas.

\section{Colaboraciones}

Claudia Bang y Marcela Corín han participado de todas las etapas de elaboración de este artículo.

\section{Agradecimientos}

A los niños, niñas y adolescentes de la aldea de Villa Soldati y sus familias, al equipo de salud del Cesac $\mathrm{N}^{\circ} 24$, a las organizaciones comunitarias, instituciones educativas, a la comunidad de artistas participantes y a los payasos terapéuticos. A los compañeros que ya no están, a los que siguen en el camino y a los que seguirán sosteniendo este proyecto colectivo que nos vincula en una ola de amor y compromiso. 


\section{Referências}

\section{ASOCIACIÓN LATINOAMERICANA DE MEDICINA}

SOCIAL (ALAMES). Documento dirigido a la

Organización Mundial de la Salud ante la Conferencia

Mundial sobre Determinantes sociales de la salud. 2011.

Disponible en: <http://www.alames.org/index.php/

documentos/declaraciones-de-la-asociacion/2011>.

Acceso em: 21 fev. 2017.

ARISTIMUÑO, S. L. et al. Miro tu esquina y veo... basura. In: CONGRESO NACIONAL DE MEDICINA GENERAL, 29., Santa Rosa, 2014.

AUGSBURGER, A. C.; GERLERO, S. S. La construcción interdisciplinaria: potencialidades para la epidemiología en salud mental. Revista de Temas Sociales, Buenos Aires, v. 9, n. 15, p. 1-10, 2015.

BANG, C. Debates y Controversias sobre el concepto de Participación Comunitaria en Salud: Una revisión histórica. Revista Eä-Humanidades Médicas \& Estudios Sociales de la Ciencia y la Tecnología, Buenos Aires, v. 3, n. 2, p. 1-23, 2011.

BANG, C. Estrategias comunitarias en promoción de salud mental: construyendo una trama conceptual para el abordaje de problemáticas psicosociales complejas. Psicoperspectivas, Valparaíso, v. 13, n. 2, p. 109-120, 2014.

BANG, C. et al. Semana de Las Crianzas en Villa Soldati: una experiencia creativa desde un Centro de Salud y Acción Comunitaria (CESAC N²4) junto a la comunidad. In: CONGRESO IBEROAMERICANO DE PRIMERA INFANCIA, 1., Buenos Aires, 2015.

\section{BERTUCELLI, S.; LERDA, M. C.; MERCADO, C. B.}

Centros de Acción Comunitaria: una nueva y antigua estrategia institucional para generar políticas sociales. Revista Iberoamericana, Rosario, v. 2, n. 3, p. 31-44, 2002.

BUSTELO, E. El Recreo de la Infancia. Buenos Aires: Siglo XXI, 2007.

CAMPOS, G. W. S. Gestión en salud: en defensa de la vida. Buenos Aires: Lugar Editorial, 2001.
CARVALHO, S. Promoción de la salud, empowerment y educación: una reflexión crítica como contribución a la reforma sanitaria. Salud Colectiva, Lanús, v. 4, n. 3, p. 335-347, 2008.

CORÍN, M. Redes y puertas en Salud Mental Comunitaria en un Centro de Salud y Acción Comunitaria (CESAC No 24 ) del sur profundo de la CABA. Revista Argentina de Psiquiatría, Buenos Aires, v. 24, p. 47-54, 2013.

\section{CHILD RIGHTS EDUCATION FOR} PROFESSIONALS (CRED-PRO). Interfase entre los derechos y la salud en la niñez: Manual de formación de Formadores. Buenos Aires: CRED-PRO, 2006.

CZERESNIA, D.; FREITAS, C. Promoción de la salud: conceptos, reflexiones y tendencias. Buenos Aires: Lugar Editorial, 2006.

FEO, O. Avanzando hacia la nueva APS: Cuidado integral de la salud con la participación de todas y todos. In: GIOVANELLA, L. (Org.). Atención Primaria de Salud en Sudamérica. Rio de Janeiro: Isags; Unasur, 2015. p. 15-22.

GEERTZ, C. El antropólogo como autor. Barcelona: Paidós, 1989.

GORODICH, R. Bordando condiciones de más dignidad. Revista Argentina de Psiquiatría, Buenos Aires, v. 14, p. 299-304, 2003.

HAMMERSLEY, M.; ATKINSON, P. Etnografía. Barcelona: Paidós, 1994.

HUANACUNI, F. Buen Vivir/Vivir Bien: Filosofía, políticas, estrategias y experiencias regionales andinas. Lima: CAOI, 2010.

MENÉNDEZ, E. Modelo de atención de los padecimientos: de exclusiones teóricas y articulaciones prácticas. Ciência \& Saúde Coletiva, Rio de Janeiro, v. 8, n. 1, p. 185-207, 2003. 
MERHY, E. Saúde: a cartografía do Trabalho Vivo. São Paulo: Hucitec, 2002.

MINAYO, M. C. S. El Desafío del Conocimiento: investigación cualitativa en salud. Buenos Aires: Lugar Editorial, 1997.

ORGANIZACIÓN PANAMERICANA DE LA SALUD

(OPS). Renovación de la Atención Primaria de la Salud en las Américas: documento de posición de la organización. Washington: OPS, 2007.

STOLKINER, A. Medicalización de la vida, sufrimiento subjetivo y prácticas en salud mental. In: LERNER, H. Los sufrimientos: 10 Psicoanalistas: 10 Enfoques. Buenos Aires: Psicolibro, 2013, p. 211-239.

STOLKINER, A.; GÓMEZ, S. A. Conceptualizando la Salud Mental en las prácticas: consideraciones desde el pensamiento de la medicina social. Revista Argentina de Psiquiatría, Buenos Aires, v. 23, p. 52-56, 2012.

VALLES, M. Técnicas cualitativas de investigación social. Madrid: Síntesis, 2000.

WEISS, R. Learning from strangers: the art and method of qualitative interview studies. New York: The Free Press, 1994

Recibido para publicación en febrero de 2017

Versión final en junio de 2017

Conflicto de intereses: inexistente

Apoyo financiero: beca posdoctoral otorgada a la primera autora por el Consejo Nacional de Investigaciones Científicas y Técnicas (Conicet), con sede en el proyecto UBACyT No 20020130100543BA del Instituto de Investigaciones de la Facultad de Psicología de la Universidad de Buenos Aires 